\title{
Simulation numérique des évolutions sédimentaires, importance d'une approche intégrée
}

\author{
Jean-Michel Hervouet, Catherine Villaret \\ Laboratoire National d'Hydraulique et Environnement. \\ Electricité De France, $R \& D$. \\ 6 Quai Watier 78400 Chatou France \\ E-mail: j-m.hervouet@edf.fr_catherine.villaret@edf.fr
}

\section{Résumé:}

Cet article illustre par des exemples l'intérêt des approches intégrée et couplée pour le calcul des évolutions sédimentaires. On y aborde, dans le cadre du système hydroinformatique Telemac, le couplage entre hydrodynamique et charriage sur le fond, le traitement simultané du charriage et de la suspension, et le calcul intégré de l'hydrodynamique et de la suspension en dimension 3.

\section{Abstract:}

This paper exemplifies, in the field of sediment transport, the need for an integrated approach coupling all physical phenomena. Three examples of combined phenomena are successively dealt with, in the framework of the Telemac hydroinformatic system: coupling of hydrodynamics with bed-load transport, simultaneous treatment of bed-load and suspended sediment transport, and integrated computation of hydrodynamics and suspended sediment in 3 dimensions.

Mots-clés : hydrodynamique, morphodynamique, charriage, suspension.

\section{Introduction}

Les évolutions sédimentaires sont la conséquence d'interactions entre la houle, les courants moyens, la turbulence, et le comportement des sédiments. Dans un domaine où les lois physiques sont insuffisamment connues, l'expertise restera toujours primordiale, mais la simulation numérique apporte des réponses de plus en plus pertinentes. Les progrès les plus récents proviennent d'une approche intégrée où tous les processus physiques sont traités simultanément, soit au sein d'un même programme, soit en ayant recours à des logiciels différents mais couplés.

Le cadre des travaux présentés ici est le système Telemac d'EDF R\&D, avec ses deux modules d'hydrodynamique: Telemac-2D (équations de Saint-Venant en dimension 2) et Telemac-3D (équations de Navier-Stokes tridimensionnelles à surface libre, avec ou sans hypothèse de pression hydrostatique, voir la référence 1), ainsi que son module sédimentaire Sisyphe (co-propriété CETMEF, UTC, EDF, SOGREAH) qui résout une équation d'évolution des fonds dans laquelle le taux de transport est calculé par différentes formules de transport par charriage. Ce modèle a été récemment étendu au transport en suspension. En dimension 2, le couplage interne, pas de temps par pas de temps, entre Telemac-2D et Sisyphe a été choisi, et son intérêt sera clairement mis en évidence dans le paragraphe 2.

L’influence de la houle est primordiale en zone littorale et a pour effets de générer des courants moyens et d'augmenter le taux de transport. Sa prise en compte passe par le couplage entre le code hydrodynamique (Telemac-2D), le modèle de propagation de la houle 
(Tomawac) et le code de morphodynamique (Sisyphe). La méthode de couplage en zone littorale fait l'objet de l'article intitulé «Modélisation du transport littoral» dans la même conférence.

Les deux modes de transport sont le charriage au niveau du fond et la suspension dans la colonne d'eau. Le premier est calculé par une formule semi-empirique, tandis que la suspension est calculée en résolvant une équation de transport-diffusion, détaillée dans le paragraphe 3. L'approche couplée charriage-suspension permet une représentation plus réaliste des écoulements hors-équilibre, comme le montre le cas de l'évolution d'une tranchée.

Dans le cas tridimensionnel, l'équation de transport-diffusion est intégrée dans Telemac3D pour modéliser la suspension, et l'on donne l'exemple dans le paragraphe 4 d'une solution analytique inspirée du profil de Rouse.

\section{$\underline{\text { 2.Couplage entre transport par charriage et hydrodynamique }}$}

\section{Approche couplée/non couplée:}

L'évolution des fonds modifie les courants, responsables de la mise en mouvement et du transport des sédiments : un couplage entre les calculs morphologique et courantologique s'avère donc indispensable dans bien des cas.

L'approche simplifiée consiste à enchaîner un premier calcul hydrodynamique avec Telemac-2D, sans tenir compte des évolutions du fond, puis à calculer ces dernières en mode permanent avec Sisyphe. La technique de correction des vitesses utilisée dans Sisyphe consiste à considérer, en mode hydrodynamique permanent, que le débit linéique (= vitesse $\mathrm{x}$ hauteur) se conserve, ce qui a pour effet, avec une mise à jour à chaque pas de temps sédimentologique, de faire croître les vitesses dans les zones de dépôt et inversement de les faire décroître dans les zones d'érosion.

Nous comparons ici cette approche simplifiée (dite 'non couplée') à une approche couplée (dite par 'couplage interne') qui consiste à calculer successivement et à chaque pas de temps hydrodynamique et morphodynamique.

Exemple 1: l'érosion autour d'un épi dans un canal. Le canal fait environ $250 \mathrm{~m}$ de large et l'épi, large de $25 \mathrm{~m}$ et long de $125 \mathrm{~m}$, en barre la moitié. La profondeur est d'environ $10 \mathrm{~m}$ et la vitesse de l'ordre de $10 \mathrm{~cm} / \mathrm{s}$. Les résultats obtenus pour un pas de temps de $2,5 \mathrm{~s}$ (et 10000 s de simulation) sont présentés sur la figure 1, avec à droite le calcul 'non couplé' (avec réactualisation simplifiée des vitesses), et à gauche le calcul avec 'couplage interne'. On constate un excès de $20 \%$ environ sur les évolutions avec le calcul 'non-couplé'. En effet, les vitesses plus fortes au passage des bosses contribuent à limiter leur croissance, phénomène qui n'est que partiellement pris en compte dans l'approche 'non couplée'. Si l'on considère simplifiée des vitesses utilisée dans Sisyphe, qui a un coût moindre, s’avère moins efficace que la version avec couplage est plus proche de la réalité, la technique de correction 


\section{Ecoulement autour d'un ouvrage dans un canal, simulation de $10000 \mathrm{~s}$} loi de transport de Meyer-Peter
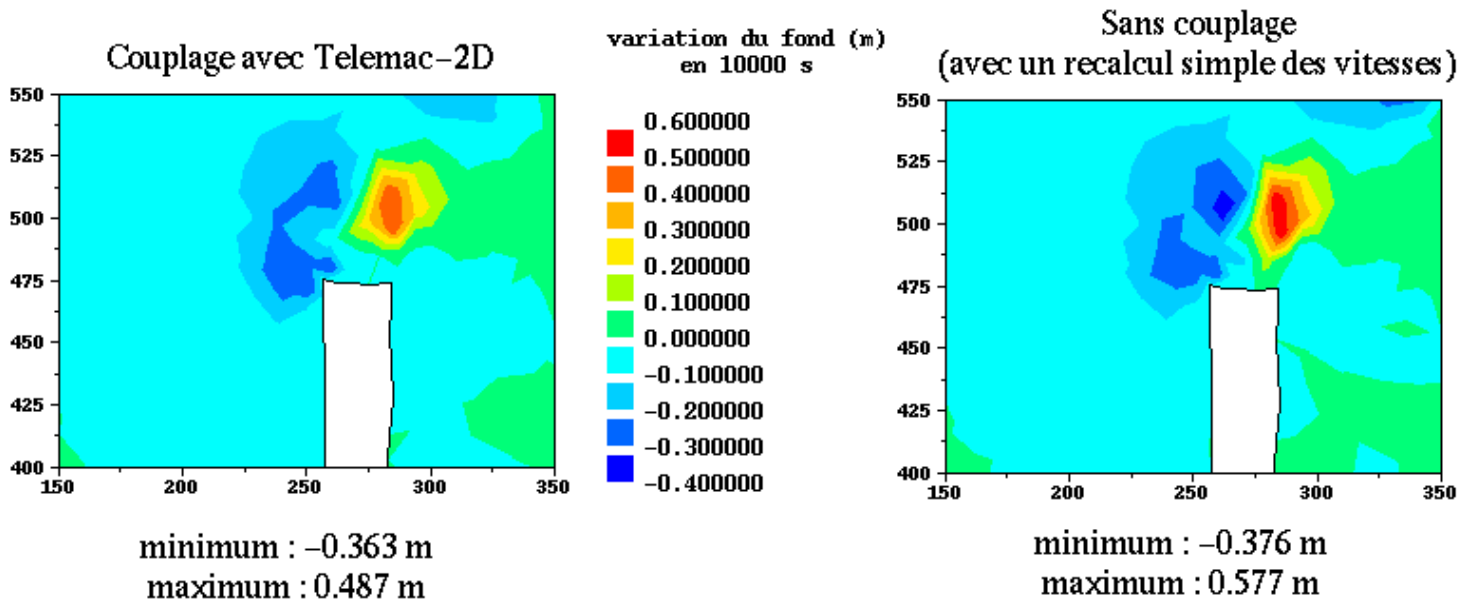

Figure 1: importance du couplage sédimentologie-hydrodynamique (coordonnées en mètres)

Exemple 2: le comblement et l'évolution d'une tranchée (profondeur : $15 \mathrm{~cm}$ et longueur : $5 \mathrm{~m})$ sous l'effet d'un courant permanent et uniforme. On se place dans les conditions expérimentales suivantes : à l'entrée hauteur d'eau de $0,5 \mathrm{~m}$, et courant moyen de $1 \mathrm{~m} / \mathrm{s}$. Les calculs couplé et non-couplé, tous autres paramètres égaux par ailleurs, sont présentés sur la figure 2. Les résultats montrent une plus grande stabilité avec le couplage interne, même si globalement on obtient la même évolution de la tranchée. Même dans ce cas simple, le couplage a pour effet de réduire les instabilités numériques. 
Topographie au temps $t=3600 \mathrm{~s}(\mathrm{~m})$

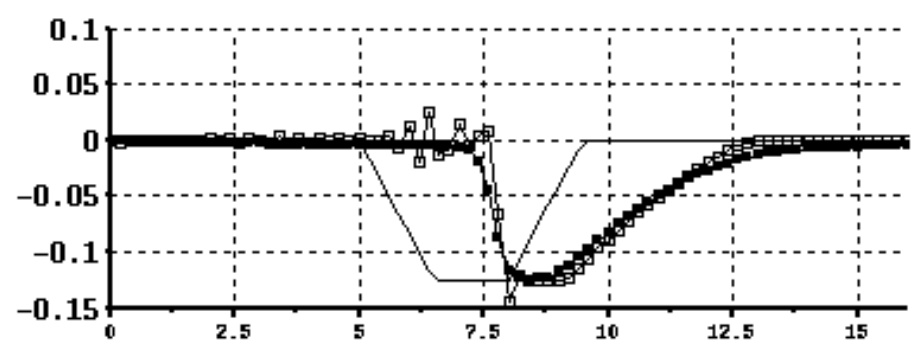

Fond initial

$\square$ Sans couplage

. Couplage Sisyphe - Telemac-2D

fond (m) au temps $t=3600 \mathrm{~s}$

0.05

0.04

$\mathbf{0 . 0 3}$

0.02

0.01

0.00

$-0.01$

$-0.02$

$-0.03$

$-0.04$

$-0.05$

$-0.06$

$-0.07$

$-0.08$

$-0.09$

$-0.10$

$-0.11$

$-0.12$

$-0.13$

$-0.14$

$-0.15$

Figure 2: Calcul du transport par charriage - Evolution d'une tranchée soumise à un courant traversier.

Conditions hydrodynamiques à l'amont : $U=1 \mathrm{~m} / \mathrm{s}, h=0,5 \mathrm{~m}$,

$$
\text { Sédiments: } D_{50}=500 \mathrm{~m}
$$

Les calculs 1 (Sisyphe seul) et 2 (Sisyphe couplé avec Telemac 2D ont été réalisés dans les mêmes conditions $(D t=0.1 \mathrm{~s}$, formule de Meyer-Peter, frottement de Strickler $\mathrm{St}=40)$ 


\section{Traitement simultané du charriage et de la suspension}

\section{Approche couplée:}

Le système Telemac était conçu à l'origine pour traiter de manière indépendante le transport en suspension (logiciel Subief 2D) et le transport par charriage (logiciel Sisyphe):

-Le logiciel Subief-2D traite le transport en suspension de matériaux fins de type cohésifs, il résout une équation de transport-diffusion pour la concentration. Sur le fond, des lois d'érosion et dépôt (Krone et Parthéniades) règlent les échanges à l'interface entre le lit et la suspension.

-Le logiciel Sisyphe traite le transport par charriage de matériaux non-cohésifs, il utilise des formulations semi-empiriques pour le taux de transport. Cette approche suppose un équilibre local entre l'écoulement turbulent et le lit de sédiments, hypothèse généralement justifiée pour le charriage. Cette approche ne permettait pas de traiter le cas général lorsque les deux composantes du transport sont du même ordre de grandeur. Avec les récents développements de Sisyphe, on traite de manière cohérente et simultanée le charriage et la suspension.

\section{Modèle conceptuel:}

La colonne d'eau est divisée en :

1)une sous-couche fine d'épaisseur à $2 \mathrm{D}_{50}$, à l'intérieur de laquelle les matériaux sont transportés par charriage; le transport par charriage s'effectue à l'équilibre.

2)le reste de la colonne d'eau où les matériaux sont transportés en suspension.

\section{Equation de transport-diffusion:}

L’hypothèse principale consiste à considérer la concentration de sédiments en suspension comme un traceur passif, i.e. qui suit l'écoulement moyen et turbulent, hormis la vitesse de chute. Par intégration sur la verticale de l'équation tridimensionnelle de transport-diffusion pour un traceur passif, de $\mathrm{z}=\mathrm{a}$ (épaisseur de la sous-couche de charriage $<<\mathrm{h}$ ) jusqu'à $\mathrm{z}=\mathrm{h}$, on aboutit alors à l'équation suivante pour la concentration moyenne en suspension $\bar{C}$ :

$$
\frac{\partial \bar{C}}{\partial t}+\bar{U} \frac{\partial \bar{C}}{\partial x}+\bar{V} \frac{\partial \bar{C}}{\partial y}=\frac{\partial}{\partial x}\left(\gamma_{t} \frac{\partial \bar{C}}{\partial x}\right)+\frac{\partial}{\partial y}\left(\gamma_{t} \frac{\partial \bar{C}}{\partial y}\right)+\frac{(E-D)_{z=a}}{h}
$$

$\gamma_{t}$ est le coefficient de diffusivité turbulente, qui intègre ici aussi un terme de dispersion dû à l'effet de moyenne sur la verticale. E et D sont les échanges à l'interface entre la suspension et la couche de charriage. L'équation d'évolution des fonds doit tenir compte de ces deux termes supplémentaires et s'écrit :

$$
(1-n) \frac{\partial Z_{f}}{\partial t}+\operatorname{div}\left(Q_{c}\right)+(E-D)_{z=a}
$$

$\mathrm{Z}_{\mathrm{f}}$ est la cote du fond, $\mathrm{n}$ la porosité et $\mathrm{Q}_{\mathrm{c}}$ le taux de transport.

\section{Flux à l'interface:}

Le terme d'échange à l'interface est $(E-D)_{z=a}=W_{c}\left(C_{e q}-C_{a}\right)$. $W_{c}$ étant la vitesse de chute des particules en suspension, $\mathrm{C}_{\text {eq }}$ la concentration à l'équilibre et $\mathrm{C}_{\mathrm{a}}$ la concentration en $\mathrm{z}=\mathrm{a}$. Lorsque l'équilibre est atteint $\left(\mathrm{C}_{\mathrm{a}}=\mathrm{C}_{\mathrm{eq}}\right)$, seul le taux de transport par charriage participe aux évolutions du fond. 


\section{Concentration à l'équilibre:}

Plusieurs formules de la littérature permettent de calculer la concentration $\mathrm{C}_{\mathrm{eq}}$ en fonction de la contrainte de frottement, dont celle de Zyserman et Fredsoe ${ }^{2}$, qui a été programmée dans Sisyphe. Il nous faut ensuite, pour connaître la concentration au fond $C_{a}$, la relier à la concentration moyenne $\bar{C}$. On suppose pour ce faire que les concentrations suivent un profil de Rouse, ce qui, allié au fait que les vitesses suivent un profil logarithmique, permet d’aboutir à la relation suivante:

$$
C_{a}=\frac{\overline{U C}}{u_{*}} \times \frac{\kappa}{I}\left[\frac{1-a / h}{a / h}\right]^{R}
$$

en supposant ici que la concentration moyenne est définie par:

$\bar{U} \bar{C}=\int_{0}^{h} U(z) C(z) d z$

avec $\mathrm{u} *$ vitesse de frottement sur le fond, $\kappa$ constante de Karman, $\mathrm{R}=\mathrm{W}_{\mathrm{c}} /\left(\kappa \mathrm{u}_{*}\right)$ où $\mathrm{W}_{\mathrm{c}}$ est la vitesse de chute (comptée positivement vers le bas) et I l'intégrale d'Einstein définie par: $I=I_{1} \log \left(33 h / k_{s}\right)+I_{2}$,

avec:

$I_{1}=\int_{a / h}^{1}\left(\frac{1-u}{u}\right)^{R} d u \quad$ et $\quad I_{2}=\int_{a / h}^{1}\left(\frac{1-u}{u}\right)^{R} \log (u) d u$

Ces intégrales sont tabulées dans Sisyphe.

\section{Application:}

Nous reprenons le cas de la tranchée du paragraphe précédent. La figure3 montre que le transport par charriage est dominant, mais que le transport en suspension n'est cependant pas négligeable, comme le prouve la figure 4, où l'on constate une accélération de l'évolution de la tranchée. La forme symétrique de la tranchée n’est pas conservée et ce phénomène est dû à la non linéarité de la formule de transport solide. Ce cas est issu du projet européen Sandpit et sa validation est en cours. On trouvera dans Celik et Rodi ${ }^{3}$ d'autres exemples.

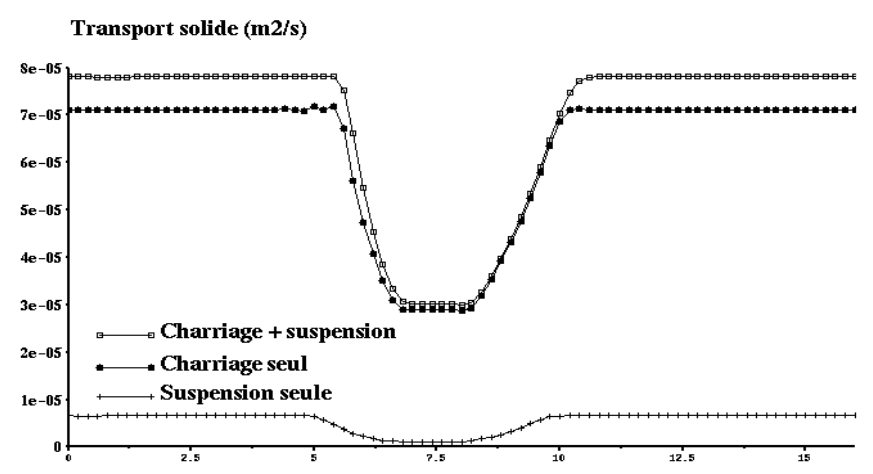

Figure 3: Couplage charriage suspension Le taux de transport total Qt est décomposé en $Q t=Q c+Q s ; Q c$ transport par charriage et Qs en suspension $(Q s=h U C)$ 


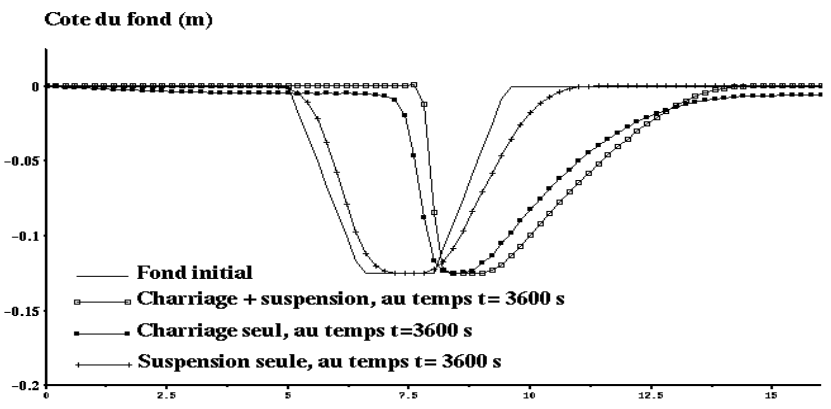

Figure 4: Couplage charriage-suspension - Evolution d'une tranchée (Les conditions de la simulation sont identiques à celles de la figure 3)

\section{Calcul intégré de l'hydrodynamique et de la suspension en 3D}

Le modèle hydrodynamique Telemac-3D est couplé à une équation de transport-diffusion pour les concentrations en suspension, qui s’écrit :

$\frac{\partial C}{\partial t}+\operatorname{div}\left[\left(\vec{U}+\vec{W}_{c}\right) C\right]=\operatorname{div}\left(\gamma_{t} \overrightarrow{\operatorname{grad}}(C)\right)$

où $\vec{W}_{c}$ est le vecteur vitesse de chute, de composantes $\left(0,0,-W_{c}\right)$. Cette équation, intégrée dans le module 3D, est donc résolue à l'intérieur de la boucle en temps, au même titre que les équations de quantité de mouvement et du modèle de turbulence.

Les résultats numériques ont été validés par comparaison avec une solution analytique, dans le cas d'un écoulement uniforme dans un canal rectangulaire à pente constante, sans frottement latéral mais avec un frottement de fond. Le modèle de longueur de mélange retenu s’écrit (voir la référence 4) :

$v_{t}=l_{m}^{2} \frac{\partial u}{\partial z} \quad$ avec $\quad l_{m}=\kappa z \sqrt{1-\frac{z}{h}}$

Ce modèle de turbulence permet d'obtenir une répartition parabolique du profil de viscosité turbulente, en accord avec le profil logarithmique des vitesses:

$v_{t}=\kappa u_{*} z\left(1-\frac{Z}{h}\right)$

Dans le cas permanent et uniforme, l'équation de transport-diffusion se simplifie pour aboutir à un équilibre sur la verticale entre les termes de diffusion et de vitesse de chute :

$\frac{\partial\left(-W_{c} C\right)}{\partial z}=\frac{\partial}{\partial z}\left(\gamma_{t} \frac{\partial C}{\partial z}\right)$

L’équation (8) s’intègre pour aboutir au profil de Rouse de la concentration :

$C(\mathrm{z})=C_{h / 2}\left[\frac{h-z}{z}\right]^{R}$ où $\mathrm{C}_{\mathrm{h} / 2}$ est la concentration à mi-hauteur $(\mathrm{z}=\mathrm{h} / 2)$

Le flux total de sédiment à l'entrée dans le canal est $\mathrm{Q} \mathrm{C}_{0}$. On peut en déduire la valeur de $\mathrm{C}_{\mathrm{h} / 2}$ par intégration du profil de Rouse. 


\section{Paramètres numériques:}

La concentration volumique des sédiments est imposée à l'amont en supposant une répartition homogène sur toute la colonne d'eau $\left(\mathrm{C}_{0}=0,02\right)$. La vitesse de chute du sédiment est de $0.01 \mathrm{~m} / \mathrm{s}$. La longueur du canal est de $500 \mathrm{~m}$, sa largeur de $100 \mathrm{~m}$, la pente du fond est de $1,011^{-3}$, afin d'obtenir, par équilibre avec le frottement, une profondeur constante de 0,5 m. Le coefficient de rugosité équivalent (loi de Nikuradse) est de 0,0162 m, qui correspond dans les conditions du calcul à un coefficient de Strickler de 50 . Le débit est $Q=50 \mathrm{~m}^{3} / \mathrm{s}$. La cote à l'aval est $0,5 \mathrm{~m}$.

Avec ces données, la profondeur dans le canal est constante, égale à $50 \mathrm{~cm}$, et la vitesse débitante de $1 \mathrm{~m} / \mathrm{s}$. Par intégration du profil logarithmique des vitesses, on peut calculer la vitesse de frottement, on trouve ici $\mathrm{u}_{*}=0,0703 \mathrm{~m} / \mathrm{s}$. Le calcul hydrodynamique avec Telemac3D est validé par une simulation de $1000 \mathrm{~s}$ avec un pas de temps de $2 \mathrm{~s}$. Le maillage contient 31 plans, c'est à dire 30 couches d'éléments sur la verticale. La différence entre théorie et calcul, aussi bien sur la viscosité que sur la vitesse, est de moins de 1\%. Pour la concentration, on trouve dans notre cas : $\mathrm{C}_{\mathrm{h} / 2}=1,8710^{-2}$. La valeur obtenue par simulation est de 1,88 $10^{-2}$. Toutefois nous avons été amenés à proposer une nouvelle solution théorique, pour rendre mieux compte du profil de concentration sur le fond.

\section{Profil de Rouse modifié:}

La solution de Rouse présente le défaut de donner une concentration infinie sur le fond, ceci est dû au fait que la viscosité turbulente y est nulle. Nous proposons donc de prendre en compte la viscosité laminaire qui n'est pas négligeable sur le fond. En supposant que $\mathrm{C}$ reste nulle en surface, nous devons maintenant résoudre:

$$
\frac{\partial\left(-W_{c} C\right)}{\partial z}=\frac{\partial}{\partial z}\left[\left(\gamma+\gamma_{t}\right) \frac{\partial C}{\partial z}\right]
$$

ce qui, quand $\frac{v}{h \kappa u^{*}}$ est petit devant 1 , s’intègre finalement pour donner :

$$
C=C_{h / 2}\left[(h-z) /\left(z+\frac{\gamma}{\kappa u^{*}}\right)\right]^{R}
$$

$\mathrm{C}_{\mathrm{h} / 2}$ doit être corrigée en conséquence et devient $1,9010^{-2}$. La viscosité laminaire évite une concentration infinie au fond. On trouve en effet $\mathrm{C}_{\mathrm{z}=0}=0,233$ pour $v=10^{-5} \mathrm{~m}^{2} / \mathrm{s}$ (valeur numérique obtenue : 0,106 ) et $\mathrm{C}_{\mathrm{z}=0}=0,106$ pour $\gamma=10^{-4} \mathrm{~m}^{2} / \mathrm{s}$ (valeur numérique obtenue : $0,0898)$. Trouver une valeur plus précise de $\mathrm{C}$ au fond nécessiterait plus de plans pour mieux représenter le profil très accentué quand $\mathrm{z}$ tend vers 0 . La figure 5 compare le profil de Rouse et le profil modifié, avec les résultats de Telemac-3D, pour $\gamma=10^{-4} \mathrm{~m}^{2} / \mathrm{s}$. Cette valeur est non réaliste mais plus démonstrative, en effet, ces résultats sont corrects pourvu que la viscosité laminaire ne soit pas trop faible, on tend sinon vers le profil de Rouse classique avec une concentration sur le fond qui tend vers l'infini. 


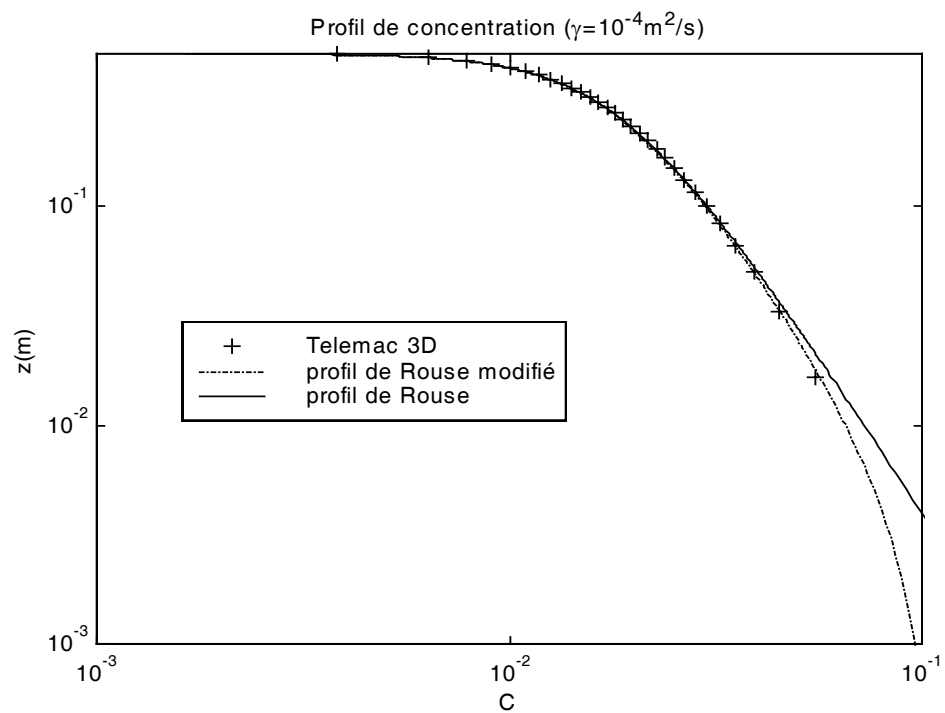

Figure 5: profil de Rouse, profil de Rouse modifié, résultats de Telemac-3D avec 31 plans sur la verticale

\section{Conclusion}

Ces quelques exemples montrent, dans des situations simples, d'une part l'importance d'un couplage sédimentologie-hydrodynamique, et d'autre part celle d'un traitement simultané du charriage et de la suspension. Enfin, une solution analytique a été proposée pour la validation du transport en suspension en dimension 3, avec la prise en compte d'une vitesse de chute. Cette solution est retrouvée par Telemac-3D avec une très bonne précision.

\section{Références}

1.Hervouet, J-M. : Hydrodynamique des écoulements à surface libre, modélisation numérique avec la méthode des éléments finis. Presses des Ponts et Chaussées. ISBN 285978-379-2. 2003

2.Zyserman, JA, Fredsoe, J. : Data analysis of bed concentration of suspended sediment, Journal of Hydraulic Engineering, ASCE, Vol. 120, N9, pp 1021-1042. 1994

3.Celik, I., Rodi , W. : Modelling Suspended Sediment Transport in non-equilibrium situations, Journal of Hydraulic Engineering, ASCE, Vol. 114, N¹0. 1988

4.Nezu, I., Nakagawa, H. : Turbulence in Open-Channel Flows, IARH, 1993 